\title{
Evaluación del estado cognitivo y afectivo en adultos mayores como estrategia de prevención
}

\section{Evaluation of cognitive and affective state in older adults as a prevention strategy}

Fecha de recepción: 20-11-2018

Fecha de aceptación: 26-04-2019
Dra. Esther Sitges Maciá

Dra. Beatriz Bonete López

Universidad Miguel Hernández de Elche.

Departamento de Psicología de la Salud

\section{resumen/alsstract:}

En el presente trabajo se presentan los resultados obtenidos de la evaluación realizada del estado cognitivo, funciones ejecutivas, estado emocional y de salud de 128 personas mayores sanas de 55 años (37 hombres y 91 mujeres) que acuden a Programas Universitarios para Mayores (PUM). El objetivo del estudio ha sido conocer el estado cognitivo, emocional y de salud de personas mayores sanas que participan en PUM y motivarles a que incorporen en sus conductas de prevención de salud revisiones periódicas en estas áreas. La media de edad de los participantes fue de 65.4 años. Los resultados mostraron que el 9.37\% de los participantes tenía algún tipo de alteración mnésica evaluada a través del MIS y un $18.75 \%$ tenía alteraciones en las funciones ejecutivas evaluadas con el Test del Reloj. Los resultados obtenidos señalan que las variables relacionadas con el estado cognitivo y la edad no son independientes, observándose una mayor proporción de personas con deterioro cognitivo en el grupo de personas de más edad. Estos resultados son concordantes con los obtenidos en estudios en este ámbito, aunque consideramos necesario seguir estudiando dicha relación en población mayor cognitivamente activa.

This paper presents the results obtained from the evaluation of the cognitive state, executive functions, emotional and health status of the 128 people over 55 years (37 men and 91 women) who attend University Programs to Older (PUM). The objective of this study was to know the cognitive, emotional and health status of the subjects, so that they incorporate this evaluation in their periodic health reviews. The average age of the participants was 65.4 years. The results showed that $9.37 \%$ of the participants had some type of alteration mnesic evaluated through the MIS and a $18.75 \%$ had alterations in the executive functions evaluated with the Test of the Clock. The results obtained indicate that the variables cognitive status and age are not independent, observing a greater proportion of people with cognitive impairment in the group of older people. These results are consistent with studies in this field, although it is necessary to continue studying such a relationship in cognitively active population.

\section{palabras clave/keywords:}

Envejecimiento activo, salud cerebral, screening cognitivo, funciones ejecutivas.

Active aging, brain health, cognitive screening, executive functions.

\section{Introducción}

Los progresos sanitarios, educativos y sociales han provocado un cambio poblacional de tal envergadura que es considerado, tal y como afirma la profesora Fernández-Ballesteros (2009), como una de las revoluciones silenciosas más importantes de la historia. Las Naciones Unidas (2017), en la revisión del informe Perspectivas de la Población Mundial, 
pusieron de manifiesto que el envejecimiento poblacional será una de las transformaciones sociales más significativas del S. XXI y este fenómeno tendrá consecuencias en todos los sectores de la sociedad, como, por ejemplo, en los servicios, la sanidad, la educación, la estructura familiar, las formas de convivencia y los lazos intergeneracionales, entre otros. En nuestro país, más del 19\% de la población tiene 65 o más años y las proyecciones indican que esta proporción de personas llegará hasta el 29\% dentro de 50 años (Abellán et al., 2019). Ante esta realidad, la Organización Mundial de la Salud (2015) ha subrayado la necesidad de promover un envejecimiento saludable instando a los diferentes países a que planifiquen acciones específicas, tales como la creación de ambientes adaptados, la adecuación del sistema sanitario a la población mayor o la mejora de la comprensión y medición del envejecimiento, entre otras. En España, a partir de la Estrategia Nacional para el Envejecimiento Activo y el Buen Trato (2017) desarrollada por el Consejo Estatal de Personas Mayores, se pretende impulsar el desarrollo y ejecución de las políticas destinadas a conseguir una mayor calidad de vida de las personas mayores y, en concreto, facilitar una vida saludable e independiente en entornos adecuados y seguros. Pensamos, al igual que indican Cardona-Arango y Peláez (2015) que las respuestas no sólo deben darse desde las instituciones, sino también desde la propia sociedad por lo que cada individuo ha de responsabilizarse de su salud a medida que envejece siguiendo el paradigma del envejecimiento activo ya que, a medida que envejece. Tal y como afirman Sánchez - Cabaco, Holgado-Sánchez, Sánchez-Zaballos y Ramos-Bernal (2014), "es también el propio sujeto que envejece agente de cambio ejerciendo un papel activo en la sociedad y viviendo una vida lo más saludable y satisfactoria posible".

Desde un enfoque multidisciplinar y biopsicosocial del envejecimiento, sabemos que las estrategias de prevención de la enfermedad y promoción de la salud han de ir encaminadas a atenuar tanto el impacto de la fragilidad derivada del proceso de envejecimiento, como la posible dependencia funcional de la persona que envejece (Abizanda, 2008; Viña y SanzRos, 2018). Y es que, las transformaciones que se producen en el proceso de envejecimiento están relacionadas tanto con factores genéticos como con las actividades que realiza la persona a lo largo de todo su ciclo vital como es la actividad física, la alimentación, o la formación académica adquirida, entre otros (Sandi, 2001). De todas, las que más preocupan a las personas mayores son aquéllas en las que está implicada la función cognitiva por su relación con patologías cognitivas degenerativas e irreversibles (Sales, Redondo, Mayordomo, Satorres-Pons y Meléndez (2016). Durante el proceso normal de envejecimiento la función cognitiva sufre un deterioro no sólo en la memoria, sino también en otros procesos como la atención y las funciones ejecutivas (Fakhri, Sikaroodi, Maleki, Oghabian y Ghanaati, 2012; Turner y Spreng, 2012) produciéndose un enlentecimiento de la velocidad de procesamiento que afecta al resto de las funciones cognitivas (Finkel y Pedersen, 2004; Bunce y Macready, 2005; Sternäng et al., 2008), aunque este enlentecimiento no sea el responsable directo de los cambios cognitivos que ocurren en el envejecimiento normal (Keys y White, 2000; Salthouse, 2010).

En el caso de las demencias, sabemos que a nivel mundial éstas afectan a unos 47.5 millones de personas y se prevé que el número total de personas con demencia pase de 75.6 
millones en 2030 a 135.5 millones en 2050 (Wortman, 2012). En la actualidad, los trabajos de investigación se han centrado en la búsqueda de marcadores tempranos que permitan predecir la demencia, tanto para su diagnóstico precoz y su prevención (García, Díaz y Peraita, 2014; Jack et al., 2018), como para identificar la relación que existe entre el deterioro cognitivo con el estado de la salud (Aparicio, Carbonell y Delgado, 2010; Brèchat, 2006), los factores genéticos o biológicos (Craik y Grady, 2002; Glisky, 2007; Salthouse, 2010; Setó-Salvia y Clarimón 2010; Sonnen et al. 2008;) o del estilo de vida para mantener una buena salud cerebral (Liu y Donaldson, 2009; Schiepers, 2018).

En la actualidad, en el ámbito universitario, nos encontramos con adultos mayores cognitivamente activos que participan en diferentes actividades culturales, formativas o de investigación ofrecidas por los Programas Universitarios para Mayores (PUM). Según los datos del último informe de la Asociación Estatal de Programas Universitarios para Mayores (AEPUM) en España, en el curso académico 2017-2018, se matricularon 58.355 alumnos en estos programas cuyo objetivo es potenciar la integración de las personas mayores activas en el contexto sociocultural que representa la Educación Superior (AEPUM, 2018). Diferentes estudios señalan que el rendimiento cognitivo de personas cognitivamente activas difiere del de aquéllas que no se involucran en actividades que requieren una activación cognitiva y, por tanto, que la implicación en este tipo de aprendizaje favorece una estimulación cognitiva continuada proporcionando efectos de protección o estrategias compensatorias que ayudarían a paliar el declive cognitivo asociado a la edad (Gázquez, Pérez-Fuentes y Carrión, 2010; González y Grasso, 2018; Labra y Menor, 2014). Un concepto relacionado con ello es la Reserva Cognitiva, "entendida como la capacidad del cerebro para afrontar y/o tolerar cambios cerebrales asociados a un envejecimiento normal o debidos a un proceso patológico, demorando o disminuyendo la sintomatología o manifestaciones clínicas" (Stern, 2002). "Es dinámica y multifactorial viéndose influida por factores como el nivel educativo o las actividades de ocio físicas, sociales y mentales realizadas (Valenzuela y Schadev, 2006; Vance y Crowe, 2006).

En la actualidad, gracias a las campañas de promoción del envejecimiento activo y saludable, son cada vez más las personas de edad avanzada que acuden de manera regular a realizarse revisiones periódicas para el control de enfermedades como el colesterol, la diabetes o la hipertensión, entre otros. Sin embargo, todavía no se ha interiorizado la importancia de realizar revisiones periódicas del estado cognitivo para conocer y actuar para retrasar, en la medida de lo posible, los síntomas de deterioro que se producen en el proceso normal de envejecimiento poniendo en marcha estrategias compensatorias. Sabemos que puede existir un amplio espacio de tiempo entre las primeras señales de inicio de algún tipo de alteración cognitiva y el diagnóstico clínico de deterioro cognitivo o demencia (Ayuso et al., 2007; Mistridis, Krumm, Monsch, Berres y Taylor (2015) por lo que el diagnóstico temprano constituye un objetivo necesario de salud pública (Villarejo y Puertas-Martín, 2011). Por todo ello, nos planteamos evaluar el estado cognitivo, emocional y de salud de una muestra de personas mayores de 55 años, cognitivamente activas que acuden a un PUM, y sensibilizarlos en la incorporación de este tipo de revisiones en sus conductas de prevención de enfermedad. 


\section{Método}

\section{Participantes}

Los participantes del presente trabajo son alumnos de Programas Universitarios para Mayores (PUM) que asistieron a las Jornadas de Puertas Abiertas realizadas dentro de la oferta de actividades complementarias que se ofrecen en los PUM de las universidades de Alicante y Miguel Hernández de Elche. Los criterios de inclusión para la selección de las personas que participaron en el estudio fueron: a) tener 55 o más años, b) deseo manifiesto de participar en la evaluación, c) funcionamiento independiente en las actividades de la vida diaria, y d) tener un nivel de alfabetización suficiente para realizar las pruebas cognitivas.

Se excluyó a los participantes que no completaron todos los test o no facilitaban datos sociodemográficos tales como la edad, estado civil, convivencia, etc. El estudio se llevó a cabo siguiendo la Declaración de Helsinki y sus modificaciones posteriores, y con las regulaciones de la Unión Europea sobre investigación médica. Los participantes no recibieron ningún reembolso financiero ni ninguna otra compensación.

\section{Instrumentos}

Los instrumentos utilizados para la evaluación fueron los siguientes:

Estado Cognitivo: Medido a través del test de cribaje Memory Impairment Screen (MIS) de Buschke et al. (1999) a través de su versión adaptada y validada al castellano de Terrón y cols. (2005). Es un instrumento breve que evalúa el recuerdo, tanto libre como facilitado con clave semántica y con una tarea distractora no semántica intercalada entre la codificación y el recuerdo. Desde la International Association of Gerontology and Geriatrics (IAGG) y el Global Aging Research Network (GRAN) indican, se trata de un test capaz de distinguir entre envejecimiento normal, deterioro cognitivo leve y demencia (Morley et al., 2015). Posee una validez semejante o superior al MMSE, destacando su rapidez y facilidad de aplicación (Villarejo y Puertas-Martín, 2011).

Funciones Ejecutivas: Han sido evaluadas a través del Test del Reloj de Cacho, García, Arcaya, Vicente y Lantada (1999). Este test evalúa las funciones visuoperceptivas, visuoconstructivas, las representaciones mentales simbólicas y las praxias. Según los autores presenta una sensibilidad del $92.8 \%$, por lo que descarta la demencia en dicho porcentaje de sujetos sanos (Cacho et al., 1999). De las dos formas de administración, a la copia y a la orden, empleamos en este estudio la segunda. Su valoración cualitativa tiene gran utilidad clínica (Villarejo y Puertas-Martín, 2011).

Estado Emocional: Por su afectación al estado cognitivo (Bastida, Pomés, Font y Eickhoff, 2016), evaluamos el estado emocional a través del test Yesavage de Depresión Geriátrica (GDS) (Yesavage y Sheik, 1986) en su adaptación española de Martínez de la Iglesia et al. (2002), utilizando la versión de 15 ítems que, según la revisión sistemática realizada por Martí et al. (2000), posee un adecuado proceso de adaptación transcultural y valores de fiabilidad superiores a 0.90. Evalúa aspectos como el afecto, la inactividad, la irritabilidad, el aislamiento, los pensamientos angustiosos y los juicios negativos. Consta de dos alternativas de respuesta dicotómica ( 
administración para el grupo de edad de personas mayores. Es un test que es ampliamente utilizado como instrumento de cribado para detectar depresión en personas mayores (Izal, Montorio, Nuevo y Pérez-Rojo, 2007).

Estado de Salud: Medimos el estado de salud general a través del cuestionario Euro Quality of Life 5-D (EQ-5D), de Badía, Roset, Montserrat, Herdman y Segura (1999). Es un instrumento que evalúa la calidad de vida relacionada con la salud de forma estandarizada y genérica. El EQ-5D consta de 2 partes: el sistema descriptivo EQ-5D y la Escala Visual Analógica (EVA). El sistema descriptivo EQ-5D comprende 5 dimensiones: movilidad, autocuidado, actividades habituales, dolor y ansiedad o depresión. En la EVA el individuo puntúa sobre el gráfico de un termómetro su salud entre dos extremos, 0 y 100, entendiendo la menor puntuación como el peor estado de saludo aumentando hasta el mejor estado de salud imaginable. En el presente estudio la variable del estado de salud se ha evaluado con ambas partes del test. En líneas generales, es un instrumento muy corto y fácil de rellenar, su repercute positivamente en la cantidad y calidad de los datos recogidos, útiles para este tipo el tipo de población con el que nos encontramos (Herdman, Badia y Berra, 2001).

\section{Procedimiento}

Una vez que los sujetos llegaban a la universidad donde se realizaban las Jornadas, antes de comenzar con las sesiones de comunicaciones y talleres, se efectuaba el screening individualizado a los asistentes por parte de los miembros del grupo de trabajo del COP-CV de la sección de Alicante. Esta evaluación tenía una duración media de entre 10 - 15 minutos por sujeto. En estas Jornadas se daba a conocer la labor del Psicólogo especialista en Envejecimiento y también tenían como objetivo concienciar, a través del screening del estado cognitivo y afectivo, las conferencias y los talleres, acerca de la importancia que tiene para desarrollar un envejecimiento activo y saludable realizar controles periódicos de estado cognitivo y afectivo y no sólo de los aspectos físicos relacionados con la salud. Al final de la Jornada, una vez corregidas las pruebas, se informaba de manera individualizada a cada persona de los resultados que había obtenido y se les recomendaba, en el caso que fuese necesario, la visita a un especialista para una evaluación neuropsicológica más exhaustiva.

\section{Análisis de datos}

Se trata de un estudio descriptivo de corte transversal de medida única. Se recogieron los datos sociodemográficos referidos al género, la edad, el estado civil, convivencia, déficit sensorial y asistencia a Programas Universitarios para Mayores (P.U.M.). Además de la recogida de las variables sociodemográficas anteriormente citadas, se evaluó el Estado Cognitivo, el Estado Emocional y el Estado de Salud General con los instrumentos anteriormente descritos. El análisis cuantitativo de los datos ha sido realizado con el paquete estadístico SPSS 23.0. Se aplicó la prueba Chi-Cuadrado para analizar la relación entre cada una de las variables dependientes (Estado de ánimo y Estado cognitivo) y el resto de variables evaluadas en el estudio. También se aplicó la prueba T-Student de muestras independientes para analizar las diferencias de medias del Estado de Salud percibido en función del resto de variables de la investigación. 


\section{Resultados}

La muestra final estuvo compuesta por 128 sujetos de los cuales la mayoría eran mujeres (91 frente a 37 hombres), con una media de edad de 65.4 años $( \pm 6.7)$. Las mujeres tenían una media de edad de 65 años, mientras que los hombres de 66.1 años. La variable Edad se categorizó en dos grupos (1=menores de 65 años y $2=$ mayores de 65 años). La muestra final quedó dividida en 63 sujetos que pertenecían al grupo 1 y 65 al grupo 2. En la Tabla 1 se muestran los resultados descriptivos en las variables sociodemográficas.

Tabla 1.- Resultados Descriptivos de las variables sociodemográficas

\begin{tabular}{lc}
\hline Variable & Porcentaje \\
\hline $\mathrm{N}=128$ & $100 \%$
\end{tabular}

Sexo

Hombres

Mujeres

\section{Estado Civil}

Soltero/a

Casado/a

Divorciado/a o Separado/a

Viudo/a

\section{Convivencia}

Sólo/a

Acompañado/a

Nivel Educativo

Sin estudios

Básicos

Medios

Superiores

\section{Déficit Sensorial}

Sin déficit sensorial

Con algún déficit sensorial

78

A la vista de los datos obtenidos en el análisis descriptivo de las pruebas utilizadas que aparecen en la Tabla 2, se aprecia que estamos ante una muestra con buen Estado de Salud Percibido, que presentan un muy buen Estado Emocional, y un elevado Estado Cognitivo. En las Funciones Ejecutivas también muestran en su mayoría una ejecución óptima de las mismas.

Desglosando los datos acerca del estado cognitivo, se observa que hay 4 personas que presentan una puntuación que podría considerarse clínica tanto en deterioro cognitivo como 
en funciones ejecutivas; 12 personas que presentaron deterioro en las funciones cognitivas generales con una puntuación menor o igual a 4 en el MIS y 22 personas con deterioro en las funciones ejecutivas con una puntuación menor o igual a 6 en el Test del Reloj. A todos ellos se les recomendó visitar a un especialista para una evaluación más exhaustiva de su estado cognitivo general. El porcentaje de personas con deterioro obtenido $(28.12 \%)$, podría ser análogo al de otros estudios realizados con poblaciones cognitivamente activas como por ejemplo el del estudio de Borson, Scanlan, Brush, Vitaliano y Dokmak (2000), quienes con una muestra de 249 personas mayores con conocimientos de varios idiomas y edades comprendidas entre 60 y 87 años, obtuvieron que un $35.6 \%$ de la muestra tenían deterioro cognitivo.

Tabla 2.- Resultados de las pruebas

\begin{tabular}{lcc}
\hline & Media & Mínimo-Máximo \\
\hline $\begin{array}{l}\text { Estado Emocional } \\
\quad \text { Yesavage }\end{array}$ & 14 & {$[0-15]$} \\
$\begin{array}{l}\text { Estado Cognitivo (Deterioro) } \\
\quad \text { Mis }\end{array}$ & 6.6 & {$[0-8]$} \\
$\begin{array}{l}\text { Funciones Ejecutivas (Deterioro) } \\
\quad \text { Test del Reloj }\end{array}$ & 8.5 & {$[0-10]$} \\
$\begin{array}{l}\text { Estado de Salud General } \\
\quad \text { Euro Quality of Life 5-D }\end{array}$ & 76.13 & {$[0-100]$} \\
\hline
\end{tabular}

Se aplicó la prueba Chi cuadrado de independencia obteniendo que no existía relación entre la variable Estado Emocional y las variables sociodemográficas Sexo $\left(X^{2} / p=0 / .98\right)$, Edad $\left(X^{2} / p=0.18 / .66\right)$, Convivencia $\left(X^{2} / p=0.61 / .43\right)$, Estado Cognitivo $\left(X^{2} / p=1.05 / .3\right), \mathrm{y}$ Funciones Ejecutivas $\left(X^{2} / p=0.19 / .65\right)$.

En la Tabla 3 se muestran los resultados del análisis de independencia de variables realizado con el estadístico Chi cuadrado entre Estado Cognitivo y las variables Sexo, Edad, Convivencia y Funciones Ejecutivas. Como se puede apreciar, es significativa a .038 la relación entre Estado Cognitivo y Edad, lo que señala la existencia de relación entre ambas.

Tabla 3.- Pruebas Chi-Cuadrado para la Variable Estado Cognitivo

\begin{tabular}{lcccc}
\hline & Sexo & Edad & Convivencia & $\begin{array}{c}\text { Funciones } \\
\text { Ejecutivas } \\
\end{array}$ \\
\hline$/ p$ & $/ p$ & $/ p$ & $/ p$ \\
\hline ESTADO & $0.049 / .825$ & $4.291 / .038^{*}$ & $1,048 / .306$ & $0,104 / .747$ \\
COGNITIVO & & & & \\
\hline
\end{tabular}


Para analizar el sentido de esta relación, se realizó una tabla de contingencia entre las variables Estado Cognitivo y Edad (Tabla 4), confirmándose que las personas de más edad son las que presentan una mayor prevalencia de deterioro del Estado Cognitivo (el 75\% de las personas con deterioro cognitivo se encuentran en el grupo de mayores de 65 años). Por ello, podemos afirmar que los resultados obtenidos para esta muestra de personas mayores de 55 años que acuden a un PUM, indican que la Edad y el Estado Cognitivo son variables dependientes existiendo una mayor proporción de personas con deterioro cognitivo en el grupo de personas de edad más avanzada.

Tabla 4.- Tabla de Contingencia de las Variables Deterioro Cognitivo y Edad.

\begin{tabular}{llccc}
\hline & & $<65$ & $>65$ & Total \\
\hline ESTADO & Sin Deterioro & 59 & 53 & 112 \\
COGNITIVO & Con Deterioro & 4 & 12 & 16 \\
TOTAL & & 63 & 65 & 128 \\
\hline
\end{tabular}

Por último, se realizó una prueba T - student para muestras independientes entre el Estado de Salud percibido y el resto de variables evaluadas y no se obtuvieron diferencias de medias estadísticamente significativas entre ellas (Tabla 5).

Tabla 5.- Análisis de diferencias de medias de Estado de Salud Percibido con T-Student

\begin{tabular}{lccccc}
\hline Variable & & N & (DT) & T & P \\
\hline Sexo & Mujer & 91 & $75.16(17.2)$ & -1.056 & .293 \\
Hombre & 37 & $78.51(13.7)$ & & \\
Edad & $>65$ años & 63 & $75.56(17.7)$ & -0.394 & .694 \\
Convivencia & $<65$ años & 65 & $76.69(15)$ & & \\
& Vive solo & 38 & $76.97(15.4)$ & 0.379 & .706 \\
& Vive & 90 & $75.78(16.7)$ & & \\
Estado Emocional & acompañado & & & & .105 \\
& Sin depresión & 121 & $76.69(16.2)$ & 1.634 & \\
Estado Cognitivo & Con depresión & 7 & $66.43(15.5)$ & & .764 \\
& Sin deterioro & 112 & $76.38(16.4)$ & 0.461 & \\
Funciones & Con deterioro & 16 & $74.38(15.8)$ & & \\
Ejecutivas & Sin deterioro & 100 & $76.35(16)$ & 0.284 & .777 \\
\hline
\end{tabular}

\section{Discusión}

Como se ha comentado anteriormente, la investigación científica sobre el deterioro cognitivo se ha centrado en la búsqueda de marcadores tempranos que permitan predecir la 
demencia, identificar la relación que existe entre el deterioro cognitivo con la salud o los factores bio-psico-sociales relacionados con el proceso normal de envejecimiento. Nuestros resultados indican que, en esta muestra, las personas de más edad son las que presentan una mayor prevalencia de deterioro del estado cognitivo. Estos resultados están en la línea de otros estudios como el reciente trabajo de Kivipelto, Mangialasche y Ngandu (2018) en el que analizan diferentes ensayos clínicos aleatorizados sobre los factores de riesgo de tener deterioro cognitivo y demencia en adultos mayores, confirmando la existencia de la relación entre la edad y la demencia.

Además de tener en cuenta la edad como factor asociado al deterioro, nos planteamos en este estudio que la incidencia de deterioro de las personas que desarrollan un envejecimiento "cognitivamente activo" como es las que participan en un PUM, sería diferente al del resto de la población. Esta hipótesis iría en la línea de los expuesto por Mas (2008) y otros trabajos que confirman que una estimulación cognitiva continuada proporciona efectos de protección o estrategias compensatorias que ayudan a paliar el declive cognitivo asociado a la edad (Gázquez, Pérez-Fuentes y Carrión, 2010; González y Grasso, 2018; Labra y Menor, 2014). El hecho de que un $28.12 \%$ de la muestra manifieste algún tipo de alteración cognitiva y que este porcentaje sea incluso mayor en otros estudios con población cognitivamente activa como el de Borson et al. (2000) confirma, por un lado, el interés que tiene este tema para la salud pública debido al incremento en nuestra sociedad de población mayor. Por otro lado, este resultado nos hace reflexionar sobre la posibilidad de que haya un infra - diagnóstico en patologías cognoscitivas en población mayor, tal y como manifiestan Villarejo et al., (2017) o la propia Sociedad Española de Neurología que afirma que entre un 30\% y un 40\% de personas se encuentran sin diagnosticar (Sociedad Española de Neurología, 2002).

Por tanto, y a la vista de los datos obtenidos, consideramos importante sensibilizar a la población mayor a realizar controles rutinarios de su estado cognitivo a medida que envejece para que, con este seguimiento, podamos conocer mejor los cambios cognitivos asociados al proceso envejecimiento y también conocer la incidencia real que tienen las alteraciones cognitivas tanto en personas que participan en actividades cognitivamente estimulantes como las que no.

Respecto a los resultados obtenidos acerca del Estado Emocional de los participantes, hemos observado que no existe una relación entre éste y el Estado Cognitivo, a pesar de algunas evidencias que existen en la literatura especializada sobre ello (Bastida, Pomés, Font y Eickhoff, 2016; Ochsner, Silvers y Buhle, 2012). Quizás nuestros resultados sean debidos al limitado número de sujetos (5) que tenían sintomatología depresiva por lo que con esta falta de variabilidad en la muestra no podemos generalizar los resultados ya que tan escaso número no nos confirma que los resultados sean fiables ni estables.

Por último podemos afirmar que se trata de un grupo de personas que se perciben con un óptimo Estado de Salud (76.13/100). Estos datos son similares a los obtenidos por Baños (2016) con una muestra de 332 alumnos de los PUM de las sedes de Burgos y Aranda que tenían una media de edad de 69.12 años y una puntuación media del Estado de Salud evaluada con el mismo instrumento de medida de 77.32. Estos resultados con muestras de 
personas que acuden a PUM son algo más altos comparados con los datos de la encuesta nacional de salud realizada por el Ministerio de Sanidad, Servicios Sociales e Igualdad en 2012 sobre la Calidad de Vida. Utilizaron también la Escala Analógica Visual (EVA) y, para el rango de adultos con edades comprendidas entre 65 y 74 años, la puntuación media fue de 60.5 para los hombres y de 48.3 para las mujeres. Que nuestros datos sean sensiblemente mayores, indica que las personas que participan en este tipo de actividades cognitivamente estimulantes como la participación en un PUM se perciben con un mejor Estado de Salud.

El tamaño muestral del presente estudio es una limitación para la extrapolación de los datos a poblaciones de similares características, por lo que continuamos en esta línea de trabajo para aumentar el número de sujetos. Asimismo, queremos replicar el estudio con una muestra de similares características sociodemográficas pero que no realicen de forma rutinaria actividades cognitivamente estimulantes y así poder evaluar los beneficios tanto para la función cognitiva como social y emocional la participación en un PUM.

En conclusión, y con las reservas comentadas anteriormente, podemos confirmar que las personas de más edad son las que proporcionalmente tienen mayor deterioro cognitivo por lo que seguimos estudiando en esta línea para conocer si el proceso de deterioro de las personas que participan en un PUM es más lento que el que se produce en personas que no son cognitivamente activas para así seguir desde estos Programas colaborando con la promoción de un envejecimiento saludable.

Agradecimientos: Queremos agradecer a todos los compañeros del Grupo de Envejecimiento del COP-CV de la sede de Alicante ya que sin su trabajo entregado y voluntarioso, este trabajo habría sido imposible de realizar.

\section{Referencias}

Abellán García, A., Aceituno Nieto, P., Pérez Díaz, J., Ramiro Fariñas, D., Ayala García, A., y Pujol Rodríguez, R. (2019). “Un perfil de las personas mayores en España, 2019. Indicadores estadísticos básicos". Madrid, Informes Envejecimiento en red, 22,38p.

Abizanda Soler, P. (2008). Fragilidad y discapacidad en el anciano. Revista Española de Geriatría y Gerontología, 43(1), 60-62.

Asociación Estatal de Programas Universitarios para Mayores. (2017-2019). Estadísticas de información del alumnado. Curso Académico 2017-2018. Recuperado de https://www.aepumayores.org/es/contenido/visor/ num_alumnos.php?idcurso=21

Aparicio García-Molina, V. A., Carbonell-Baeza, A., y Delgado Fernández, M. (2010). Beneficios de la actividad física en personas mayores. Revista Internacional de Medicina y Ciencias de la Actividad Física y del Deporte, (40), 4-20.

Ayuso, T., Ederra, M. J., Manubens, J. M., Nuin, M. A., Villar, D., y Zubicoa, J. (2007). Abordaje de la demencia. Guía de actuación en la coordinación Atención Primaria-Neurología. Servicio Navarro de Salud: Osasunbidea.

Badia, X., Roset, M., Montserrat, S., Herdman, M., y Segura, A. (1999). The Spanish version of Euro0ol: a description and its applications. European Quality of Life scale. Medicina clínica, 112, 79-85.

Baños, V. (2016). Aplicación del cuestionario de salud Eq-5d-5I en alumnado mayor universitario. International Journal of Developmental and Educational Psychology, 1(2), 307-316. 
Bastida, J. D., Pomés, N. P., Font, S. J., y Eickhoff, A. F. (2016). La depresión: un predictor de demencia. Revista Española de Geriatría y Gerontología, 51(2), 112-118.

Borson, S., Scanlan, J., Brush, M., Vitaliano, P., y Dokmak, A. (2000). The mini cog: a cognitive 'vital signs' measure for dementia screening in multi lingual elderly. International journal of geriatric psychiatry, 15(11), 1021-1027.

Bréchat, P. H., Lonsdorfer, J., Berthel, M., y Bertrand, D. (2006). Subsidising exercise in elderly people. The Lancet, 367(9516), 1055-1056.

Bunce, D., y Macready, A. (2005). Processing speed, executive function, and age differences in remembering and knowing. The Quarterly Journal of Experimental Psychology Section A, 58(1), 155-168.

Buschke, H., Kuslansky, G., Katz, M., Stewart, W. F., Sliwinski, M. J., Eckholdt, H. M., y Lipton, R. B. (1999). Screening for dementia with the memory impairment screen. Neurology, 52(2), 231-231.

Cacho, J., García-García, R., Arcaya, J., Vicente, J. L., y Lantada, N. (1999). Una propuesta de aplicación y puntuación del test del reloj en la enfermedad de Alzheimer. Revista de Neurología, 28(7), 648-655.

Cardona-Arango, D., y Peláez, E. (2015). Envejecimiento poblacional en el siglo XXI: oportunidades, retos y preocupaciones. Salud Uninort, 28(2), 335-348.

Craik, F. I., y Grady, C. L. (2002). Aging, memory, and frontal lobe functioning. New York, NY, US: Oxford University Press.

De Pedro-Cuesta, J., Virués-Ortega, J., Vega, S., Seijo-Martínez, M., Saz, P., Rodríguez, F., ... y Martínez-Martín, P. (2009). Prevalence of dementia and major dementia subtypes in Spanish populations: a reanalysis of dementia prevalence surveys, 1990-2008. BMC. Neurology, 9(1), 55.

Delgado Vergara, T., y Pereira Pérez, J. (2017). El envejecimiento: un fenómeno demográfico con repercusión jurídica. Revista Novedades en Población, 13(26), 24-39.

Fakhri, M., Sikaroodi, H., Maleki, F., Ali Oghabian, M., y Ghanaati, H. (2012). Age-related frontal hyperactivation observed across different working memory tasks: an fMRI study. Behavioural neurology, 25(4), 351-361.

Fernández-Ballesteros, R. (2009). Envejecimiento activo: Contribuciones de la psicología. Madrid: Pirámide.

Finkel, D., y Pedersen, N. L. (2004). Processing speed and longitudinal trajectories of change for cognitive abilities: The Swedish Adoption/Twin Study of Aging. Aging Neuropsychology and Cognition, 11(2-3), 325-345.

García-Herranz, S., Díaz-Mardomingo, M. C., y Peraita, H. (2014). Evaluación y seguimiento del envejecimiento sano y con deterioro cognitivo leve (DCL) a través del TAVEC. Anales de Psicología, 30(1), 372-379.

Gázquez, J.J., Pérez-Fuentes, Ma.C. y Carrión, J.J. (2010). Análisis de la Memoria Cotidiana en alumnos del Programa Universitario para Mayores en Almería. European Journal of Education and Psychology, 3(1), 155-165.

Glisky, E. L. (2007). Changes in cognitive function in human aging. En D. R. Riddle (Ed.), Brain Aging: Models, Methods, and Mechanisms. Boca Raton (FL): CRC Press/Taylor \& Francis.

González, M.J. y Grasso, L. (2018). Plasticidad cognitiva en el envejecimiento exitoso, aportes desde la evaluación del potencial de aprendizaje. Estudios de Psicología, 39(2-3), 337-353.

Herdman, M., Badía, X., y Berra, S. (2001). El EuroQol-5D: una alternativa sencilla para la medición de la calidad de vida relacionada con la salud en atención primaria. Atención primaria, 28(6), 425-429.

Izal, M., Montorio, I., Nuevo, R., y Pérez-Rojo, G. (2007). Comparación de la sensibilidad y la especificidad entre diferentes versiones de la Escala de Depresión Geriátrica. Revista española de geriatría y gerontología, 42(4), 227-232.

Jack, C. R., Bennett, D. A., Blennow, K., Carrillo, M. C., Dunn, B., Haeberlein, S. B., ... y Liu, E. (2018). NIA-AA Research Framework: Toward a biological definition of Alzheimer's disease. Alzheimer's \& Dementia, 14(4), 535562.

Keys, B. A., y White, D. A. (2000). Exploring the relationship between age, executive abilities, and psychomotor speed. Journal of the International Neuropsychological Society, 6(1), 76-82.

Kivipelto, M., Mangialasche, F., y Ngandu, T. (2018). Lifestyle interventions to prevent cognitive impairment, dementia and Alzheimer disease. Nature Reviews Neurology, 14(1), 653-666.

Labra, J.A., y Menor, J. (2014). Estimulación cotidiana y funcionamiento cognitivo: la importancia de la participación de personas mayores sanas en actividades cotidianas cognitivamente demandantes. European Journal of Investigation in Health, Psychology and Education, 4(3), 309-319. 
Martí, D., Miralles, R., Llorach, I., García-Palleiro, P., Esperanza, A., Guillén, J., y Cervera, A. (2000). Trastornos depresivos en una unidad de convalecencia: experiencia y validación de una versión española de 15 preguntas de la escala de depresión geriátrica de Yesavage. Revista Española de Geriatría y Gerontología, 35(1), 7-14.

Martínez de La Iglesia, J., Onís Vilches, M., Dueñas Herrero, R., Albert Colomer, C., Aguado Taberné, C., y Luque Luque, R. (2002). Versión española del cuestionario de Yesavage abreviado (GDS) para el despistaje de depresión en mayores de 65 años: adaptación y validación. Medifam, 12(10), 26-40.

Mas, C. (2008). Mejora de la memoria en personas mayores: variables a tener en cuenta. Papeles del Psicólogo, 29(2), 213-221.

Ministerio de Sanidad, Servicios Sociales e Igualdad. Madrid (2012). Encuesta Nacional de Salud. España 2011/12. Serie Informes monográficos $\mathrm{n}^{0} 3$. Calidad de vida relacionada con la salud en adultos: E0- 5D-5L.

Mistridis, P., Krumm, S., Monsch, A. U., Berres, M., y Taylor, K. I. (2015). The 12 years preceding mild cognitive impairment due to Alzheimer's disease: the temporal emergence of cognitive decline. Journal of Alzheimer's Disease, 48(4), 1095-1107.

Morley, J. E., Morris, J. C., Berg-Weger, M., Borson, S., Carpenter, B. D., del Campo, N., ... y Ganguli, M. (2015). Brain health: The importance of recognizing cognitive impairment: An IAGG consensus conference. Journal of the American Medical Directors Association, 16(9), 731-739.

Nieto, M.A. (2011). Cambios cognitivos en el envejecimiento normal: un estudio de seguimiento. Memoria final del proyecto 59/2011 del Ministerio de Sanidad, Servicios Sociales e Igualdad.

Ochsner, K. N., Silvers, J. A., y Buhle, J. T. (2012). Functional imaging studies of emotion regulation: a synthetic review and evolving model of the cognitive control of emotion. Annals of the New York Academy of Sciences, $1251(1), 1-24$.

Organización Mundial de la Salud (2015). Informe Mundial sobre el Envejecimiento y la Salud. OMS: Estados Unidos de América. Recuperado 12/3/2019 en: https://apps.who.int/iris/bitstream/handle/10665/186466/9789240694873_ spa.pdf;jsessionid=957A85295B7B7B31C41C161C97E887A7? sequence $=1$

Organización Mundial de la Salud (2016). 69ª Asamblea Mundial. Documento 69/17: Acción Multisectorial para un envejecimiento saludable basado en el ciclo de vida: proyecto de estrategia y plan de acción mundiales sobre el envejecimiento y la salud. Recuperado 12/3/2019 en: http://apps.who.int/gb/ebwha/pdf_files/WHA69/A69_17sp.pdf

Rodríguez, V. R., Manas, L. R., Castiello, M. S., y Martín, R. D. (2012). Envejecimiento. La investigación en España y Europa. Revista Española de Geriatría y Gerontología, 47(4), 174-179.

Sales, A., Redondo, R., Mayordomo, T., Satorres-Pons, E., y Meléndez, J.C. (2016) Diferencias entre personas mayores sanas y con deterioro cognitivo leve en variables clínicas. Psicogeriatría, 6(2), 61-67.

Salthouse, T. A. (2010). Selective review of cognitive aging. Journal of the International Neuropsychological Society, 16(5), 754-760.

Sánchez-Cabaco, A., Holgado Sánchez, A., Sánchez Zaballos, E., y Ramos Bernal, M. T. (2014). Año europeo. envejecimiento activo y solidaridad intergeneracional. International Journal of Developmental and Educational Psychology. Revista INFAD de Psicología, 7(1), 533-540.

Sandi, C., Venero, C., y Cordero, M. I. (2001). Estrés, memoria y trastornos asociados: Implicaciones en el daño cerebral y el envejecimiento. Ariel.

Schiepers, O. J., Köhler, S., Deckers, K., Irving, K., O'donnell, C. A., van den Akker, M., ... y van Boxtel, M. P. (2018). Lifestyle for Brain Health (LIBRA): a new model for dementia prevention. International journal of geriatric psychiatry, 33(1), 167-175.

Sociedad Española de Neurología (2002). Propuesta de criterios para el diagnóstico clínico del deterioro cognitivo ligero, la demencia y la enfermedad de Alzheimer. Neurología 17(1), 17-32.

Setó-Salvia, N., y Clarimón, J. (2010). Genética en la enfermedad de Alzheimer. Revista de Neurología, 50(6), 360-364.

Sitges Maciá, E. y Bonete López, B. (2015). Desarrollo de un programa psicoeducativo en inteligencia emocional para cuidadores principales de enfermos de Alzheimer. European Journal of Investigation in Health, Psychology and Education, 4(3), 257-266. 
Sonnen, J. A., Montine, K. S., Quinn, J. F., Kaye, J. A., Breitner, J. C., y Montine, T. J. (2008). Biomarkers for cognitive impairment and dementia in elderly people. The Lancet Neurology, 7(8), 704-714.

Stern, Y. (2002). What is cognitive reserve? Theory and research application of the reserve concept. Journal of the International Neuropsychological Society, 8(3),448-460

Sternäng, 0., Wahlin, A., y Nilsson, L. G. (2008). Examination of the processing speed account in a population based longitudinal study with narrow age cohort design. Scandinavian journal of psychology, 49(5), 419-428.

Terrón, C., Peña-Casanova, J., Manero, R., Böhm, P., Gramunt, N. y S. Quiñones. (2005). Versión española del Memory Impairment Screen (MIS): datos normativos y de validez discriminativa. Neurología, 20(8), 402-411.

Turner, G. R., y Spreng, R. N. (2012). Executive functions and neurocognitive aging: dissociable patterns of brain activity. Neurobiology of aging, 33(4), 826-831.

Valenzuela, M. y Sachdev, P. (2006). Brain reserve and dementia: A systematic review. Psychological Medicine, 36(4), 441-454.

Vance, D. E. y Crowe, M. (2006). A proposed model of neuroplasticity and cognitive reserve in older adults. Activities, Adaptation \& Aging, 30(3), 61-79.

Villarejo, A., y Puertas-Martín, V. (2011). Utilidad de los test breves en el cribado de demencia. Neurología, 26(7), 425433.

Villarejo, A., Eimil, M., Llamas, S., Llanero, M., López de Silanes, C. y Prieto (2017). Informe de la Fundación del Cerebro. Impacto social de la enfermedad de Alzheimer y otras demencias. Neurología, 2017.

Viña, J., y Sanz-Ros, J. (2018). Alzheimer's disease: Only prevention makes sense. European Journal of Clinical Investigation, 48(10), e13005.

Wight, R.G., Aneshensel, C.S. y Seeman, T.E. (2002). Educational Attainment, Continued Learning Experience, and Cognitive Function among Older Men. Journal of Aging and Health, 14(2), 211-236.

Wortmann, M. (2012). Dementia: a global health priority-highlights from an ADI and World Health Organization report. Alzheimer's research \& therapy, 4(5), 4-40.

Yesavage, J. A., y Sheikh, J. I. (1986). 9/Geriatric depression scale (GDS) recent evidence and development of a shorter version. Clinical gerontologist, 5(1-2), 165-173. 